\title{
CLAUDIO ESTEVA FABREGAT, NOVATOR DE LA CIENCIA DE LA CULTURA EN ESPAÑA
}

\author{
Luis Calvo-Calvo \\ Institución Milá y Fontanals \\ Consejo Superior de Investigaciones Científicas \\ Icalvo@imf.csic.es
}

\section{A modo de introducción}

Cada una de las revoluciones científicas que se han producido a lo largo de la historia ha propiciado nuevos avances; así, los planteamientos de Francis Bacon (1561-1626) hicieron que tanto el método inductivo como la organización y la especialización se convirtiesen en claves de bóveda de la investigación científica. Por su importancia, estas aportaciones le merecieron la consideración de novator.

Desde mi punto de vista, la obra antropológica - conceptual y formalmente- de Claudio Esteva Fabregat puede ser definida como la propia de un novator: desde su regreso a España, aunque de manera concreta, desde su asentamiento en Barcelona a partir de 1968, su objetivo fue dotar a la antropología cultural de un estatus y de una fortaleza que le permitiese significarse como disciplina propia y diferenciada. Tal como él mismo declaró en una ocasión, otros estudiosos, como Julio Caro Baroja, realizaron aportaciones más que significativas al conocimiento antropológico, pero 
sus contribuciones se llevaron a cabo fuera del ámbito universitario, por lo que no tuvieron suficiente repercusión ni contribuyeron a consolidar e institucionalizar la antropología en España.

Sobre este periodo de la vida profesional de Esteva ya existen diversos escritos, incluso del propio autor (Esteva, 1982; Prat y Martínez, 1996; Calvo, 1997; Capel, 2009; Lagunas, 2012; y Calvo, 2017). Por ello, este texto intenta profundizar en algunas de las claves de sus primeros años en Barcelona, cuando sus esfuerzos se concentraron especialmente en disponer los cimientos, tanto en lo institucional como en lo profesional, que permitieron, con el tiempo, hacer de la antropología cultural una disciplina consolidada en la Universidad y fuera de ella.

\section{Esteva y la ciencia de la cultura}

Constituir la ciencia de la cultura como disciplina claramente diferencia$\mathrm{da}$, con personalidad propia en lo formal y en lo conceptual, fue el principal interés de Esteva cuando llegó a Barcelona. La actuación que realizó cabe inscribirla en su propia concepción de la antropología como materia autónoma. De esta manera, todos sus esfuerzos desde su llegada a la Universidad de Barcelona (UB) y al Consejo Superior de Investigaciones Científicas (CSIC) en Barcelona iban dirigidos a reforzar su enfoque respecto de la antropología. A pesar de la extensión de la cita, en ella refleja su pensamiento sobre qué se debe entender por antropología:

Quan parlem d'Antropologia, no em sembla propi d'aquesta integrar-la dintre el concepte de ciència social, sinó que li és més adient considerar-la una ciencia natural de la cultura. En la tradició, som naturalistes perquè [...] hem estat associats amb el treball de camp i amb qüestions, com l'evolucionisme humà, de què hem tractat juntament amb els antropòlegs físics i els prehistoriadors; però també amb els materials dels etòlegs, els paleontòlegs, els geògrafs, els geòlegs [...] Pensem, a més, que l'home és un animal social, com ho són altres espècies. Ens en distingim, però, per un fet diferenciador cabdal: la cultura [...] som naturalistes de l'estudi de la cultura [...] donat aquest caràcter específic, podem optar pel concepte de ciència cultural, o de ciència humana, mentre que 
no escau dir ciència social perquè aquesta definició no és la més apropiada per als continguts estratègics del que estudiem. Per la meva part, prefereixo, el de ciència cultural [...] (Esteva, 2008: 32).

Asimismo, en una entrevista a principios de la década de 1980, Esteva declaró: «[...] crec que l'home no es pot estudiar de manera parcial perquè l'home és un individu que comprèn tota una sèrie de situacions, una més propiament biològica, una altra que és històrica, una altra de contemporània» (Hernández, 1982: 45).

Esta noción de lo que debía ser la antropología, en buena medida, en clara conexión con la visión norteamericana de la disciplina, fue el principal acicate de las labores de Esteva, con vistas a la consolidación de la materia como entidad universitaria independiente, más allá de la tutela que otras disciplinas como la prehistoria y la arqueología habían ejercido históricamente sobre los estudios antropológicos en España.

Esteva, con el objetivo de diseñar la implantación de la antropología a imitación del modelo boasiano, esto es, según la perspectiva de que esta disciplina ha de abarcar la antropología biológica, la etnología, la arqueología y la lingüística, estableció un conjunto de actuaciones dirigidas desde la UB y el Centro de Etnología Peninsular -e Hispanoamericana, como añadirían más tarde- $(\mathrm{CEPH})$ del CSIC. Estas medidas, con el tiempo, posibilitaron que la antropología cultural adquiriese rango académico en paridad con otras disciplinas humanísticas similares. De entre aquellas medidas, posiblemente, el ejemplo más sobresaliente fue el propio diseño del I Congreso Español de Antropología, celebrado en Barcelona entre el 28 marzo y el 2 de abril de 1977. La amplitud de las cuestiones tratadas puso de manifiesto la idea holística de Esteva; así, el encuentro se articuló a través de los siguientes ejes temáticos: 1) Relaciones interdisciplinarias. 2) Las sociedades campesinas en España. 3) Historia de la Antropología. 4) Etnohistoria. 5) Arqueología americana. 6) Antropología biológica (VV. AA., 1980).

Su visión de la antropología, diferente de la que hasta su llegada a España imperaba, se manifestó muy tempranamente cuando ya en 1957, en Madrid y como respuesta a la pregunta «¿Qué conclusiones pueden conseguirse con el análisis comparativo de la cultura?», escribía lo siguiente: 
«Fundamentalmente, establecer la validez relativa de cada respuesta cultural en términos de tiempo y espacio, poner en relación y clasificación histórica a los pueblos y poner en evidencia aquellos procesos que llevan al concepto de leyes socio-culturales» ${ }^{1}$.

Estas aseveraciones nos recuerdan el fuerte apego de Esteva por todo aquello que hacía referencia al estudio y profundización en la teoría antropológica, hecho que también se puso de manifiesto nada más llegar a España. Así, como miembro de la delegación española $-\mathrm{y}$, en concreto, del Seminario de Estudios Americanistas de la Universidad de Madridque participó en el VI Congreso Internacional de Ciencias Antropológicas y Etnológicas, celebrado en París entre el 30 de julio y el 6 de agosto de 1960, presentó una ponencia titulada «El método funcionalista en el estudio de la aculturación», donde puso de manifiesto, entre otras cosas, su visión acerca de ciertos problemas que siempre lo preocuparon, como es el caso de los procesos de aculturación ${ }^{2}$. En este sentido, planteaba lo siguiente: «[...] el antropólogo funcionalista no puede reducir su campo de trabajo al estudio de la estructura y actividades de una agregación fenotípica de formas culturales. También deberá mostrar el proceso por el cual se reúnen las actividades dentro de los propósitos sociales internos [...] la situación diacrónica característica de todo proceso de cambio socio-cultural debe estudiarse actuando sobre los puntos donde se ha interrumpido la trama asociativa tradicional y, asimismo, reconociendo dónde se efectúa el nuevo equilibrio de la acción social» (Esteva, 1964: 586).

Esteva se mantuvo fiel a su idea culturalista; con tal objetivo, fijó claramente su ideario: «[...] antropología cultural i, per tant, un aspecte històric molt pronunciat, un interés centrat en l'estudi dels elements de cultura, de les formes culturals. Aquí és on en moc més», concepción que se contraponía a la antropología social, la cual, según Esteva, está orientada «[...] sociològicament. És un tipus d'investigació de camp que es desenvolupa dintre d'una tradició sociològica i l'única diferencia que acostuma a tenir amb els sociòlegs és que fan més el que se'n diu treball de camp i

I Fondo documental de la Institución Milá y Fontanals (CSIC, Barcelona).

2 Su interés por este tema siempre lo manifestó hasta el punto de decir lo siguiente: «En realitat tinc com a preocupació fonamental [...] els problemes de l'aculturació, ésa dir, els problemes del canvi de cultura en poblacions que estan en contacte unes amb les altres» (Hernández, 1982: 48). 
elaboren també més el model etnogràfic, mentre que el sociòleg normalment no ho fa, sinó que, per mitjà de l'enquesta, localitza els models concrets i acostuma a quantificar-los i té una tècnica diferent. La influència d'aquesta antropología social ve donada bàsicament per una influència britànica» (Hernández, 1982: 50). Para Esteva, la principal diferencia entre la antropología cultural y la social era que esta última: «[...] no se ocupa de sistemas, cosa que sí se propone hacer el holismo etnográfico y, sobre todo, el análisis de lo que es superorgánico» (Esteva, 2008: 59).

De esta forma, para Esteva, la antropología cultural era una: «[...] ciencia que se ocupa del estudio comparado de las culturas y de las costumbres humanas en su evolución y adaptaciones en el tiempo y el espacio [siendo] su objetivo el conocimiento de las leyes que rigen el proceso histórico de las formas culturales en sus variedades ecológicas y su transmisión y transformaciones» (Esteva, 1993: 26).

Al hilo de estas consideraciones, Esteva siempre defendió que el término central que articulase toda su actuación científica e institucional fuese el de antropología cultural, ya que consideró que «[...] en gran medida, además, era indispensable propiciar el desarrollo de una concepción teórica inclusiva más amplia y la Antropología Cultural cumplía estos requisitos [...] siguiendo un desarrollo con precedentes en las tradiciones antropológicas de los EE. UU. e Iberoamérica, [por lo tanto,] tenía que designarse con el nombre de Antropología Cultural» (Esteva, 1982: 16).

A modo de conclusión de este apartado, cabe señalar que con su llegada a Barcelona pudo, junto a su denodada preocupación por asentar la disciplina en la Universidad, desarrollar su obra científica. Así, sus principales obras vieron la luz y se constituyeron en su momento en referentes por la novedad de las temáticas tratadas, así como por su fuerte acento teórico (Esteva, 1973b; Esteva, 1975; Esteva, 1978; y Esteva, 1984a). Todas estas obras pusieron de manifiesto la relevancia de la antropología cultural como disciplina en una sociedad y una academia - y al servicio de ellas - que necesitaban de cambios perentorios en muchos órdenes. En relación con su producción científica y con la perspectiva que otorga el tiempo, posiblemente la pregunta que hoy cabría hacerse es: ¿en qué medida estas obras han sido referentes para la propia disciplina y en nuestras universidades? 


\section{Hacia la institucionalización de la antropología: el contexto}

Como el propio Esteva ha explicado en diversas ocasiones, así como otros autores han puesto de manifiesto (véanse las referencias mencionadas en las primeras líneas de este artículo), su principal interés al llegar a la UB fue propiciar todo aquello que permitiese que la antropología cultural se erigiese en ciencia propia en paridad con otras disciplinas humanísticas y sociales. Esta tarea no era sencilla por diversas razones, ya que el contexto en el que se tenía que llevar a cabo esta actuación no era, realmente, el más propicio.

En este sentido, cabe recordar que Esteva empezó sus labores docentes e investigadoras en el ámbito de la prehistoria. Así, en el curso 1967196832, Prehistoria y Etnología eran materias en el primer curso de la licenciatura general de Historia, así como en la licenciatura de Historia Antigua. Las impartían Luis Pericot, alumno y heredero de Pere Bosch i Gimpera en la UB -quien, a pesar de su interés por la etnología, no contaba con formación antropológica específica-, y August Panyella, a la sazón, director del Museo Etnológico de Barcelona (MEB) ${ }^{4}$. Ello conllevó que la etnología se considerase subsidiaria de la prehistoria. Entre otras cosas, esta circunstancia comportó que, cuando se crearon los departamentos en la Facultad de Filosofía y Letras (Orden Ministerial de 28 de 1966, BOE de julio), el Departamento de Prehistoria —dependiente de la Sección de Historia, dirigida por Pericot- dispensara las siguientes asignaturas: Prehistoria, Arqueología, Epigrafía y Numismática, Historia de Grecia y Roma, Historia Social y Económica del Mundo Antiguo, Etnología, América Prehispánica e Historia Antigua, Universal y de España. De esta forma, en aquellos momentos, el único acercamiento posible a la etnología - que no antropología cultural- en Barcelona era la que Panyella ofrecía mediante su labor museística y docente en el MEB y en sus clases en la $\mathrm{UB}^{5}$. En este sentido, tal como se ha venido sugiriendo

\footnotetext{
3 Vid. las páginas 63-64 del Anuario de la Universidad de Barcelona 1967-1968. Barcelona: Universidad de Barcelona.

$4 \mathrm{Al}$ respecto, vid. la página 43 del Anuario de la Universidad de Barcelona de 1961-1962, donde se observa que Panyella, como profesor encargado de curso, impartía Etnología en la Sección de Historia.

5 Hasta que Esteva consiguió la agregaduría en 1968, Panyella era profesor adjunto (provisional) y profesor encargado de curso. Vid. las páginas 37 y 39 del Anuario de la Universidad de Barcelona 1968-
} 
más arriba, la concepción de Panyella todavía implicaba en gran medida que la biología era la fuente principal de la cultura. Así se desprendía de la memoria que el propio Panyella presentó al Ayuntamiento de Barcelona para conseguir la pertinente autorización con el objetivo de realizar una expedición a Etiopía, la cual se llevó a cabo en los meses de enero y febrero de 1974: «[...] una expedición etnológico-antropológica a Etiopía, dado el extraordinario interés raciológico, cultural y religioso de este país [...] a) Interés excepcional de la zona del Macizo de Abisinia como centro de la dispersión de la raza etiópica, núcleo raciológico de transición entre európidos y melánidos. b) Zona de confluencia de lenguas y culturas de origen cuchita (camita) y semita. c) Confluencia de religiones precristianas, cristianismo, judaísmo y mahometismo. d) Gran tradición político-cultural y artesanía» ${ }^{6}$.

El fuerte vínculo al componente biológico también quedó reflejado en el cuaderno de viaje de Panyella cuando, en el Aeropuerto de Roma (17 de enero de 1974), anotó lo siguiente: «Viaja un interesante subgrupo — creo filipinos - antropológicamente 'polimorfos': 1 paleo mongol, una proto-malaya, de raíz de nariz hundida, mentón huidizo, labios gruesos, 1 al parecer híbrido de tagalo + español, 1 levemente negroide de piel —acaso igorrote- de rasgos algo primitivos» ${ }^{7}$.

Asimismo, en el ámbito de los estudios folklóricos, la noción que se tenía de la antropología era claramente evolucionista, decimonónica, y se concebían las supervivencias culturales como expresión de la cultura primigenia, sin aceptar que el cambio o el proceso son elementos conformadores de las investigaciones. De esta forma, y a modo de ejemplo, se creía en la «[...] mayor aptitud del medio femenino para conservar y transmitir folklore. Las mujeres se fijan mucho en los detalles nimios, se los cuentan unas a otras y saben explicarse mejor que los hombres [... se]

\footnotetext{
1969. Barcelona: Universidad de Barcelona. En el año académico de 1968-1969, Panyella impartía Etnología en el primer curso de la licenciatura de Historia Antigua durante 3 horas a la semana (ibidem, página 68). Cabe señalar que en este mismo año, entre las materias de libre elección, aparecía una Antropología impartida por el doctor Sitges en la Sección de Biológicas (ibidem).

6 Vid. el fondo documental del MEB: Expedición a Etiopía (1974).

7 Ibidem.
} 
aconseja interrogar en primer término a los ancianos que son los buzones de alcance del pasado» (Casas, 1947: 265; la cursiva es mía).

Por otra parte, otro polo universitario donde se impartía Antropología era en la licenciatura de Ciencias, Sección de Biológicas, que en su quinto curso ofrecía esta materia, impartida por el catedrático Santiago Alcobé, quien la enseñaba desde la década de $1940^{8}$.

En última instancia, no cabe dejar de lado el propio contexto universitario en el que, por poner algún ejemplo, se convalidaban los estudios eclesiásticos ${ }^{9}$ o había ayudas para los «españoles repatriados de Rusia» ${ }^{10}$.

Por lo tanto, la primera tarea de Esteva cuando llegó a la UB fue gestionar el cambio de paradigma con respecto a la etnología. En primera instancia, se propuso que se asimilase a la antropología cultural como una parte del proceso de estudio, análisis e interpretación de la ciencia antropológica, es decir, debía concebirse como una parte de esta (etnografía, etnología, antropología). En este sentido, Esteva consideró que la etnología histórica, es decir, la asociada a arcaísmos y primitivismos culturales - por asociación con las investigaciones de los ámbitos de la prehistoria y la arqueología-, no había llegado a producir ni investigaciones suficientemente sólidas ni teorías propias sobre lo primitivo y, por ello, la etnografía que se había practicado hasta aquel momento solamente había tenido como finalidad aportar datos que complementasen las investigaciones en los ámbitos mencionados. Por todo ello, la etnología había devenido en una disciplina meramente auxiliar (véase Esteva, 2008: 41-53). El propio Esteva ya indicó en un texto de 1969 las deficiencias que para la antropología como ciencia conllevaba esta visión: métodos descriptivos, autodidactismo y baja profesionalización, ausencia de financiación y falta de planes de estudio (Esteva, 1969; citado en Prat, 1992: 21). De esta forma, al poco de llegar a la UB, Esteva ya pudo introducir

8 Vid. la páginas 89 del Anuario de la Universidad de Barcelona 1967-1968. Barcelona: Universidad de Barcelona. Al igual que en la Sección de Historia, en 1966 se creó en la Sección de Biológicas el Departamento de Antropología, dirigido por el mismo Alcobé, que incluía las siguientes dos materias: Antropología e Historia de las Ciencias Naturales y Afines (ibidem, página 92). También existían cursos de Antropología en los estudios de Filosofía, impartidos por F. Gomá, pero estos, como los orientados a la biología, se obvian en este trabajo.

9 Ibidem, páginas 156-159.

10 Ibidem, páginas 169-170. 
nuevos conceptos en los estudios de su especialización; así, en el curso 1969-1970 se incluyeron nuevas materias como Antropología Cultural, Etnología de los Pueblos Primitivos o Arqueología Americana, materias impartidas por el propio Esteva y por Josefina Roma, miembro del Departamento de Prehistoria ${ }^{11}$. De manera progresiva, se fueron introduciendo nuevas materias, como Cultura Andina, que se unieron a la llegada de nuevos profesores, como Joan Frigolé, para el curso $1971-1972^{12}$, aunque todo ello tenía lugar todavía en el seno del Departamento de Prehistoria e Historia Antigua, hasta la constitución formal del Departamento de Antropología Cultural. Cuando este ya estuvo constituido formalmente, uno de los principales logros fue que en los nuevos planes experimentales de la universidad, en el seno de la nueva Facultad de Geografía e Historia, Antropología Cultural fuese materia obligatoria de primer curso, lo que supuso un avance significativo en el acercamiento de la materia a centenares de estudiantes (recuérdese que solamente en el curso 1975-1976 la referida facultad tenía unos 3000 alumnos), así como un progresivo aumento del profesorado ${ }^{13}$. Asimismo, si se observan las asignaturas de carácter antropológico de los planes de estudios del curso académico de 1977-1978, se puede apreciar el rápido crecimiento de la disciplina; así, junto a la ya comentada asignatura de Antropología Cultural en el primer curso, en segundo y tercero se impartían Etnología Americana, Etnología de los Países Catalanes, Etnología de los Pueblos Primitivos, Etnología de la Península Ibérica y Antropología Económica y Social. Con respecto al segundo ciclo, en cuarto y quinto se enseñaba Teoría e Histo-

11 Véanse las páginas 69-70 del Anuario de la Universidad de Barcelona 1969-1970. Barcelona: Universidad de Barcelona.

12 Vid. las páginas 196, 209 y 235 del Anuario de la Universidad de Barcelona 1971-1972. Barcelona: Universidad de Barcelona.

13 Así, en el curso 1975-1976 ya eran profesores agregados o encargados de curso los doctores M. J. Buxó, J. Contreras y Joan Prat. La extensión de la UB en otras ciudades, como Tarragona, comportó que allí también se iniciasen las enseñanzas antropológicas con profesores como J. J. Pujadas. Vid. las páginas 15, 17 y 28 del apéndice del Anuario de la Universidad de Barcelona 1975-1976. Barcelona: Universidad de Barcelona. Lo mismo ocurrió en Lleida (vid. las páginas 106-107 del Anuario de la Universidad de Barcelona 1977-1978. Barcelona: Universidad de Barcelona. 
ria de la Antropología Cultural, Cultura y Personalidad, Aculturación y Cambio Social, Mitología, Magia y Religión, y Antropología cognitiva ${ }^{14}$.

El progresivo desarrollo de la antropología cultural rompió, finalmente, la dependencia con la prehistoria, tradición fuertemente arraigada en el contexto académico español. Solo cabe ver, en este sentido, el programa de enseñanza de la Universitat Autònoma de Barcelona del curso 19361937, en el que se aprecia la referida subordinación de la etnología que, anclada en la tradición, se concebía exclusivamente como un elemento auxiliar de las ciencias del pasado:

SECCIÓ D'HISTORIES. Cicles d'Història antiga, medieval i moderna. Agrupaments: I. Etnologia. Prehistòria general. Història d'Orient. Història d'Europa en l'antiguitat. Institucions gregues i romanes [...] Cicle d'Arxius, biblioteques i museus. Museus. Agrupaments: I. Etnologia. Prehistoria general. Pràctiques de Prehistòria. Prehistòria de la Península Ibèrica. Arqueologia oriental. Arqueologia clàssica. Arqueologia clàssica de la Península Ibèrica. Numismàtica antiga. Epigrafia grega i llatina [...] SECCIÓ D’HISTÒRIES. Cicle d'Història antiga. ETNOLOGIA. Lluís Pericot. Problemes generals del'Etnologia [..] Seminaris. ETNOLOGIA. Lluís Pericot. Treballs per a un catàleg de tribus sudamericanes $^{15}$.

Cuando Esteva se incorporó a lo largo de 1968 al Departamento de Prehistoria e Historia Antigua de la $\mathrm{UB}^{16}$ tras ganar el concurso para ocupar un puesto de agregado, su principal tarea consistió en poner en valor los conceptos básicos y propios de la ciencia antropológica. Con este objetivo, puso énfasis en aspectos como la teoría y la comparación cultural, por lo que, cuando se hizo cargo de la enseñanza de materias como Etnología de los Pueblos Primitivos, su principal interés radicó en que la etnología dejase de ser subsidiaria de la prehistoria y mostró otros puntos de vista

14 Vid. las páginas 100-102 del Anuario de la Universidad de Barcelona 1977-1978. Barcelona: Universidad de Barcelona.

15 Universitat Autònoma de Barcelona. Facultat de Filosofia i Lletres i Pedagogia. Programa de cursos per l'any 1936-1937.

16 La primera referencia de Esteva en la UB como profesor agregado aparece en la página 32 del Anuario de la Universidad de Barcelona 1968-1969. Barcelona: Universidad de Barcelona. Asimismo, Esteva pasó a impartir Etnología en el primer año de la licenciatura de Historia Antigua. A partir de este anuario, Panyella dejó de aparecer en el listado de profesores. 
sobre los pueblos ágrafos que los alejaba del primitivismo cultural e intentaba presentar otras visiones de sus vidas y modos de organización; todo ello desde perspectivas claramente holísticas. De esta forma, Esteva se propuso romper con los discursos y las argumentaciones de carácter diacrónico para introducir la sincronía en el análisis de los mal llamados pueblos primitivos. Así, a la hora de hablar de estos o de las culturas prehispánicas americanas, pretendió que lo fundamental fuese presentar «[...] la función y [el] proceso simultáneos de la vida social, en organizaciones de tiempo y espacios horizontales» (Esteva, 2008: 49; vg. economía, organización social, arte, religión, personalidad).

En resumen, Esteva pretendía que la etnología se concibiera como una teoría de la etnografía comparada y, por ende, que la antropología cultural respondiera a una teoría de la etnología comparada (Esteva, 2008).

\section{Esteva Fabregat y el reto de la institucionalización de la antropología}

Tal como escribió Carmelo Lisón Tolosana respecto de los propósitos de Esteva: «[...] su meta fundamental [fue] la institucionalización universitaria de la antropología» (Lisón, 2010: 34).

Por ello, cuando Esteva llegó a la UB en 1968 se planteó que su tarea más urgente era iniciar un proceso de consolidación progresiva de la disciplina, una vez liberada de sus históricas dependencias. Así, consideró que la única manera era «[...] crear l'escola, i després, la mateixa gent que sortís d'aquesta escola es constituiría en un grup de pressió i provocaria l'existència de possibilitats perquè hi hagués un reconeixement oficial» (Hernández, 1982: 47), intención que siempre tuvo presente en su actuación desde aquel lejano 1968: «Desde luego, pensábamos que la tarea no podía progresar sin una previa institucionalización académica de la Antropología Cultural, cosa que por entonces [...] no se había logrado convenientemente. Por esta razón, era indispensable profundizar antes en la vida académica como condición indispensable [...]» (Esteva, 1984b: 3). 
De esta forma, consideró que la fuerte ligazón, en lo formal y en lo conceptual, de la etnología con la prehistoria impedía que la antropología alcanzase el estatus que merecía.

En concreto, ese reconocimiento oficial se obtuvo una vez que consiguió la cátedra en 1971 y a raíz de la creación del Departamento del Antropología Cultural en 1972, fruto de una actuación concertada entre la UB y el CSIC —en concreto, del $\mathrm{CEPH}^{17}$-, del que Esteva consiguió dotaciones económicas, aunque siempre escasas en relación con las necesidades, para conseguir sus objetivos. Esteva siempre insistió en la necesidad de obtener fondos económicos suficientes, ya que sin ellos no podía lograr sus planes: «[...] los recursos fueron escasos desde el primer momento, y tuvimos que confrontar un plan de prioridades opcionales. En las condiciones de penuria informativa con que se movía la entonces incipiente academia antropológica, llegamos a la conclusión de que los pequeños recursos de que disponíamos debían emplearse en la creación de una biblioteca que, partiendo de cero, pudiera convertirse en un centro de información para estudiosos de la Antropología y, en particular, de la Etnología y de la Antropología Social» (Esteva, 1984b).

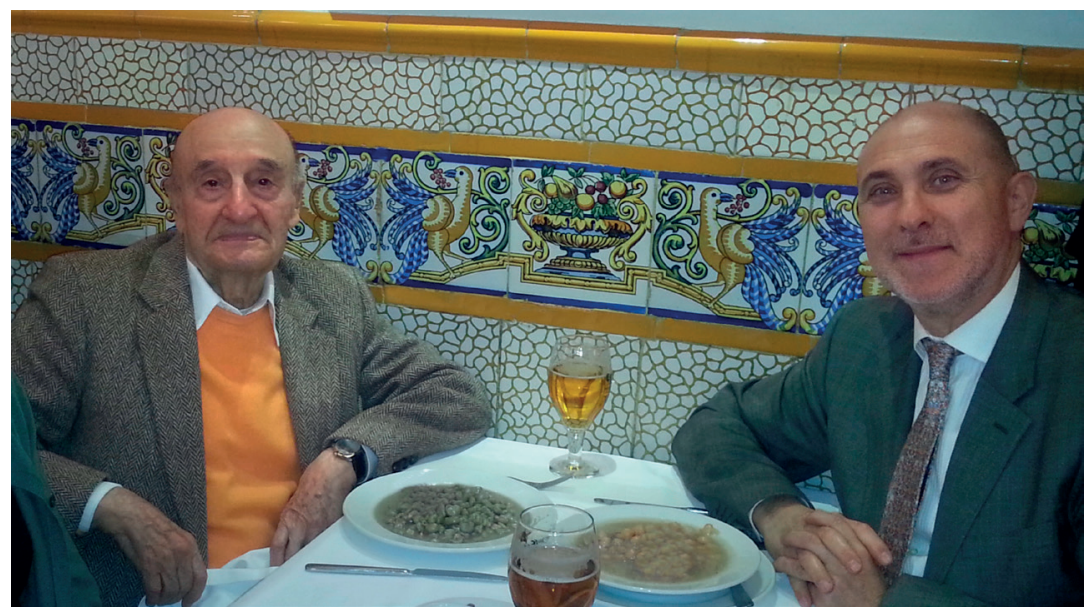

Claudi Esteva Fabregat i Lluis Calvo. Barcelona 2015 @ Berta Alcañiz.

17 Tal como se ha mencionado más arriba, a finales de la década de 1970 se añadió «e Hispanoamericana» con el objetivo de ampliar la base investigadora del centro. Por ello, el acrónimo aquí usado es el de CEPH. 
En el caso de la Universidad, Esteva empezó a poner en marcha paulatinamente un plan de estudios que fuese ampliando el campo formal y conceptual de la disciplina, tal como se ha indicado más arriba. Al unísono, en el ámbito del CSIC llevó a cabo una estrategia en tres direcciones: formación, creación de un fondo bibliográfico de referencia y divulgación de las actividades antropológicas: «[...] esto fue lo que ocurrió: la Universidad implantó sus cursos y planes de estudio y el CEPH se ocupó de llenar un primer vacío [...] formó una biblioteca para especialistas acreditados, con sus correspondientes dotaciones de revistas y libros que permitieran atender a las necesidades de información que son indispensables para el desarrollo de una disciplina de amplio espectro, como lo es, en este caso, la Antropología» (Esteva, 1984b).

Cabe destacar esta estrategia que permitió, con el paso del tiempo, consolidar la antropología como disciplina en paridad con el resto de las ciencias humanísticas. De esta forma, las memorias del CEPH permiten conocer de manera más concreta la precitada estrategia. Así, ya en la memoria del centro correspondiente a 1970 se decía: «Este Centro, sirve también a la formación del universitario que desea especializarse en Antropología, y, por tanto, una buena parte de los esfuerzos estarán dedicados a proporcionar ocasiones a los alumnos, de realizar prácticas en trabajos de campo, así como contribuir a su formación mediante una biblioteca especializada» ${ }^{18}$. De esta manera, Esteva puso especial énfasis en que los estudiantes se formasen de la manera más sólida posible en la teoría y en la práctica antropológicas, ya que el CEPH era «[...] una prolongación de los esfuerzos docentes de la Cátedra de Antropología Cultural de la Facultad de Filosofía y Letras de Barcelona» ${ }^{19}$.

Así, las claves de actuación del CEPH incluían la formación especializada, la constitución de un fondo bibliográfico y la difusión a través de la creación de una publicación periódica, como fue Ethnica, «[...] cuyo objeto es publicar trabajos de investigación en Antropología, tanto nacionales como extranjeros, que estudien temas en los que el Centro está interesado» ${ }^{20}$.

18 Memoria del Centro de Etnología Peninsular (1970). Fondo documental IMF-CSIC.

19 Centro de Etnología Peninsular. Respuesta al Comité Ejecutivo de Investigación Científica e Investigación Universitaria del CSIC. Fondo documental IMF-CSIC.

$20 \mathrm{Ibidem}$. 
A pesar de que todas estas acciones perseguían el mismo objetivo, posiblemente el aspecto formativo fue el que más bien reflejó la impronta que Esteva quería imprimir al CEPH y, por ende, a su labor. De esta manera, dicha formación pasaba, básicamente, por «[...] la aplicación del enfoque etnográfico, es decir, la observación directa y personal de los fenómenos que son objeto de estudio» (Frigolé, 1971: 232). Por ello, el aprendizaje de todo lo que suponía el trabajo de campo fue, realmente, eje de las preocupaciones y quehaceres de Esteva, ya que su concepción de la antropología lo llevaba a considerar que la investigación antropológica debía ser «[...] dinámica y empírica, ya que la teoría sigue a los datos» (Frigolé, 1971). Así, ya en 1972 y según la visión holística de Esteva, el CEPH «[...] desarrolla su actividad investigadora en dos campos: el trabajo de Archivo, y el Trabajo de Campo» ${ }^{21}$.

Por ello, buena parte de los esfuerzos de Esteva en el CEPH los encauzó a conseguir recursos para el correcto aprendizaje de las técnicas antropológicas a través de estudios y trabajos de campo, ya fuesen en la propia Península o en América del Sur; así, en el primer caso y a modo de ejemplo, Esteva y los primeros alumnos focalizaron su atención en el Alto Aragón, donde quisieron «[...] obtener materiales sobre aculturación y cambio social en el Alto Aragón» (Frigolé, 1971: 228; Esteva, 1971; y Comas / Pujades, 1975). En el segundo caso, Esteva, gracias a su participación en la Misión Científica Española en Perú, pudo trabajar en Chinchero con algunos de sus estudiantes barceloneses (véase Contreras, 1996), lo que contribuyó a su formación antropológica a través, por ejemplo, del «estudio del carácter social del chincherino, así como se ha investigado intensivamente en relación con la medicina popular, brujería y curanderismo. Además, se ha procurado completar materiales relativos al sistema de propiedad y a la organización económica de las diferentes comunidades que forman parte del distrito de Chinchero» ${ }^{22}$.

Esta concepción de la antropología permitió a Esteva dotar a la disciplina de rasgos singulares y diferenciales con respecto a las disciplinas a las que había estado supeditada hasta entonces: conceptos como cambio,

21 Documento del CEPH para el Comité Ejecutivo de Investigación Científica e Investigación Universitaria del CSIC (12 de noviembre de 1972). Fondo documental IMF-CSIC.

22 Memoria CEPH-CSIC 1970. Fondo documental IMF-CSIC. 
proceso, conflicto o aculturación se convertían en aquel momento en nuevos referentes. Cabe resaltar que el contexto en que se incorporaban ideas como las mencionadas era complejo ya que, de una parte, aquellas se oponían a las tendencias intelectuales y académicas predominantes en la década de los años setenta en la Universidad española, como el mar$x_{i s m o}{ }^{23}, y$, de otra, suponía romper el vínculo, fuerte e, intelectualmente hablando, casi opresivo, que mantenían la prehistoria y la arqueología clásicas con la etnología. A pesar de ello, no se puede olvidar que incorporar conceptos como los expresados a la vida académica e intelectual suponía, entre otras cosas, contribuir de manera decidida a labrar nuevos caminos, en la España de aquellos momentos, que supusiesen reconocer la diversidad social, política y cultural, así como abrir nuevos horizontes democráticos para la propia Universidad y para la sociedad en general.

Tanto en la UB como en el CEPH, la labor no fue sencilla, ya que la falta de recursos se suplía con voluntarismo por parte de todos los participantes del momento; así, en una petición de Esteva de octubre de 1974 al CSIC se decía lo siguiente: «En las actuales condiciones es ya difícil incorporar personal con carácter gratuito para desempeñar las actividades que son propias de este Centro, y en este sentido, a menudo nos vemos obligados a mantener cerrados los servicios e instalaciones del CEP a causa de la imposibilidad de contar con personal permanente. En estas circunstancias, debo expresar que nuestra situación, en cuanto a la regularidad de funcionamiento del Centro de Etnología Peninsular es prácticamente difícil de continuar» ${ }^{24}$.

A pesar de ello, ante el requerimiento del CSIC para presentar programas al IV Plan de Desarrollo y la posibilidad de que el CEPH pudiese convertirse en un centro de investigación nacional, Esteva y sus colaboradores, de manera especial, Josefina Roma, prepararon un ambicioso programa de investigación que llevó por título «Estudio antropológico de las zonas agrarias deprimidas y en trance de despoblamiento y de las grandes ciudades receptoras», que tenía por objetivos «la promoción de

23 A modo de ejemplo, véase el artículo de Esteva en que reflexionaba sobre la idoneidad de usar unos u otros términos para tratar aspectos de aculturación (Esteva, 1973a).

24 Carta de Claudio Esteva Fabregat al presidente del patronato Saavedra Fajardo (4 de octubre de 1974). Fondo documental IMF-CSIC. 
las grandes zonas agrarias deprimidas para que pudiesen ser autosuficientes y productoras [, y, en segundo término] para agilizar las ciudades, liberándolas de la masificación y la despersonalización de sus relaciones» ${ }^{25}$.

Esta iniciativa sintetizaba, en buena medida, algunas de las aspiraciones, formales y conceptuales, de Esteva con relación a dos hechos: en primer lugar, la necesidad de focalizar nuevos objetos de estudio, como las migraciones o el mundo urbano; en segundo término, quería insistir en la dimensión aplicada del trabajo antropológico porque «[...] el antropólogo es quien, en todo el mundo, desde el sector más desarrollado como en programas norteamericanos o europeos, hasta los países del Tercer Mundo, se encarga de los estudios previos a la aplicación de medidas económicas. Esto ocurre, porque el antropólogo es el especialista en el estudio de pueblos, de agrupamientos y sociedades, de instituciones y costumbres. De evolución, y de cambio ante un fenómeno llegado del exterior. De actitudes y sistema de valores [...] el antropólogo no trabaja desde su despacho, sino que estudia las sociedades dentro de ellas. $\mathrm{Su}$ conocimiento es cualitativo en gran parte y está en lo posible desligado de los prejuicios que en ocasiones provoca el trabajo superficial, cuantitativo y de despacho. Su información, por tanto, es más de fiar que la de otro especialista» ${ }^{26}$.

Asimismo, y a pesar de las dificultades para dar forma a sus ideas, Esteva no cejó en proponer nuevas perspectivas como, por ejemplo, todo lo referido al estudio del mundo urbano; de esta manera, planteó la necesidad de considerar la ciudad de Barcelona como laboratorio de estudio, ya que estimaba que la metodología etnográfica podía aportar al estudio del mundo urbano «[...] una perspectiva casi inédita en las tradiciones de nuestras disciplinas [que] puede proporcionarnos claves importantes para el entendimiento de los fenómenos de la cultura moderna» ${ }^{27}$. Su interés por este nuevo campo de estudio residía en que consideraba que

25 Extraído del programa de investigación propuesto por el Centro de Etnología Peninsular para el IV Plan de Desarrollo. Fondo documental IMF-CSIC.

26 Ibidem.

27 «Memoria de las actividades del Centro de Etnología Peninsular (1970-1972), de Barcelona». Ethnica 5 (Barcelona, 1973), p. 218. 
las dinámicas de las ciudades «[...] afectan no sólo al hombre urbano sino que también su influencia llega a los centros rurales de una manera que convierte a éstos en una función de las ciudades [...] hoy no podemos determinar claramente si un campesino es absolutamente campesino, pues lo que realmente ocurre con su dependencia del mercado urbano es que, en realidad, es ya una variable del mundo de las ciudades ${ }^{28}$. Cabe destacar que cuando Esteva se refería a esa «perspectiva casi inédita», estaba constatando la necesidad de que la antropología se implicase hasta sus últimas consecuencias en la contemporaneidad, por lo que planteaba un cambio de paradigma y, para ello, proponía concebir «[...] la etnografía rural desde el punto de vista de las fuerzas dinámicas que la condicionan [...] porque el mundo, en su proceso de urbanización creciente, es ya una función de las ideologías urbanas» ${ }^{29}$.

Aunque estas iniciativas no se pudieron llevar a cabo - recuérdese que es el momento histórico del tardofranquismo, así como por las propias circunstancias del $\mathrm{CEPH}$-, las labores coordinadas del Departamento de Antropología Cultural de la UB y del centro del CSIC dieron sus frutos, sobre todo en lo referido a la formación de la primera generación de antropólogos en la $\mathrm{UB}^{30}$ y a la edición, desde 1971 hasta 1984, de Ethnica. Revista de Antropología - aunque las dificultades hicieron, a modo de ejemplo, que la publicación solo contara con un volumen anual a partir de 1977, con excepción del volumen 18, de 1982, del que se publicaron dos fascículos- (véase Calvo, 1996; recogido también en Prat / Martínez, 1996: 42-49; una versión ampliada de este texto se puede consultar en Calvo, 2002a).

El esfuerzo por darle continuidad a Ethnica fue notable y rápidamente la publicación se hizo hueco en el panorama antropológico nacional e

28 Ibid., pp. 218-219.

29 Ibid., p. 219. Uno de los resultados más destacados de esta investigación se recogió en un texto de Esteva Fabregat dedicado a la aculturación (Esteva, 1973a).

30 Así, se redactaron las primeras tesis de licenciatura en antropología; entre otras, Josefina Roma, Los A-ni'to y su función en la sociedad Igorrote de Filipinas; Joan Frigolé, Estudio histórico-sociológico de la vila de Bañolas en el año 1900; Jesús Contreras, Empirismo y superstición en la medicina popular y en la medicina académica del siglo xviII; Dolores Soriano, Aspecto socioeconómico de un pueblo del Pirineo: Campellas; María Jesús Buxó, Etnografía del habla: modelos de relación diádica en Catalunya; Montserrat Camps, Tópicos y estructura social; Joan Prat, Estudio de los cuentos infantiles; Manuel Moreno, Essai sur le Don. 
internacional (vid. Calvo, 2002b; Jiménez / Checa, 2012; y Prat, 1999). Ello quedó acreditado tanto por los autores, consagrados (Bosch i Gimpera, Mead, Zamora, Alcina Franch, Valls, Esteva) o noveles (Buxó, Contreras, Frigolé, Moreno, Pujadas, Roma, Terrades...) como por los intercambios y las suscripciones, de manera especial las de carácter internacional, ya fuesen privadas o institucionales ${ }^{31}$. De esta manera, Ethnica se convirtió en el mejor ejemplo del cambio que estaba viviendo la antropología española.

En otras ocasiones ya se ha comentado que el proceso de consolidación institucional de la antropología en España no estuvo exento de dificultades y, como ha ocurrido en otros ámbitos y disciplinas en transcursos similares, la perspectiva del tiempo permite aquilatar mucho mejor algunas actuaciones y decisiones. Así, el propio Esteva era consciente, desde la asunción en 1968 de la dirección del debilitado CEPH del CSIC, al que incorporó el antiguo Archivo de Etnografía y Folklore de Cataluña, que también languidecía en el CSIC, de que los recursos humanos y económicos eran esenciales para alcanzar las tareas fundamentales ya mencionadas (formación, constitución de un fondo bibliográfico, difusión). En este sentido, hay que destacar que Esteva lideraba el proyecto, pero otras personas que trabajaron junto a él fueron fundamentales para que el CEPH y Ethnica adquiriesen el prestigio del que llegaron a gozar. Así, en una primera época, Josefina Roma asumió todo lo referido a la secretaría del centro y de la revista y, años más tarde, María Jesús Buxó; en los últimos años de Esteva en el CSIC hicieron lo propio Berta Alcañiz y un joven estudiante que hoy tiene la oportunidad de firmar el presente texto.

Tal como se ha apuntado líneas atrás, Esteva luchó por conseguir fondos económicos del CSIC para el centro; de esta manera, consiguió un presupuesto de 350000 pesetas anuales para aquel y 200000 pese-

31 A modo de ejemplo, en el trienio 1983-1985, algunas de las entidades que estaban suscritas o que mantenían intercambios con Ethnica fueron las siguientes: Institut für Völkerkunde der Universität zu Köln (Alemania), Göteborg Etnografiska Museum (Suecia), Associazione Italiana Studi Americanistici (Italia), Universidade dos Açores (Portugal), Royal Anthropological Institute, Peabody Museum, Bodleian Library-Oxford (Reino Unido), Instituto de Investigaciones Antropológicas (México), Centro de Investigaciones Regionales de Mesoamérica (Guatemala), Fundación Ameghino (Argentina), Fondo de Promoción de la Cultura Popular (Colombia), Universidad Católica de Tachira (Venezuela), The Library of Congress, Indiana University, Boston University, Harvard University, Arizona State University (EE. UU.), University of Alberta (Canadá), Kinokuniya Company (Japón). 
tas para la edición de Ethnica, así como otros fondos adicionales para, por ejemplo, realizar los trabajos de campo durante la Misión Científica Española en Perú (50 000 pesetas) o los realizados en el Pirineo catalán y aragonés, así como para el acondicionamiento material del centro. Si en el ámbito científico pronto empezaron a obtenerse resultados fehacientes - como la realización de tesis doctorales-, en el caso de la revista Ethnica, su éxito fue inmediato: no solo publicaba trabajos propios, sino también de antropólogos ya consolidados en la escena internacional, tal como se ha mencionado más arriba.

El hecho de que, a partir de un determinado momento, hacia 1978, el CEPH viviese diferentes fracturas internas propició que en 1984 el centro desapareciese y, con él, Ethnica dejara de publicarse. Con relación a su desaparición, Esteva indicó que el final de la publicación se debió a la falta de apoyo del CSIC. Posiblemente, fueran dos las razones últimas del final de la publicación: la reestructuración que el CSIC llevó a cabo con el fin de constituirse en un organismo autónomo de investigación, sin la tutela de los catedráticos de universidad; y el hecho de que el CEPH había perdido buena parte de su potencial investigador y científico debido a las mencionadas fracturas internas. Así las cosas, desde finales de la década de 1970 hasta 1985 aproximadamente, las actuaciones del CEPH decayeron significativamente, aunque cierta actividad formativa externa continuó hasta el curso académico 1985-198632.

Con la perspectiva que ofrece el paso de los años, se puede decir que el cierre del centro y el final de Ethnica fueron pérdidas significativas en el contexto de una joven disciplina que estaba abriéndose camino en el ámbito académico, nacional y foráneo: Ethnica fue, posiblemente, el mejor embajador de la antropología española y, solamente con el tiempo y a raíz de cambios sucesivos, publicaciones como la Revista de Dialectologíay Tradiciones Populares, la Revista d'Etnologia de Catalunya, el Arxiu d'Etnografia de Catalunya o la Gazeta de Antropología han seguido la estela de aquella.

32 Como ejemplo, destacan algunos de los cursos externos que se impartieron en los últimos años del CEPH en 1983: Antropología, Biología y Cultura, Antropología de los Grupos Marginales de la Península Ibérica, Antropología de las Culturas Mediterráneas, España y los Indios del Suroeste de Estados Unidos, Antropología del Mito; y en 1985, el de Algonquinos e Iroqueses de los Bosques de Norteamérica. 
En última instancia, cuando la primera generación de antropólogos formados con Esteva está entrando ya en la jubilación y después de los muchos avatares de la disciplina desde aquellos tiempos fundacionales (véanse los cambios en los planes de estudio), cabe preguntarse si el diseño de la disciplina que se realizó en aquellos años hoy sigue siendo válido $\mathrm{y}$, sobre todo, si las nuevas generaciones de antropólogos están creando «[...] diseños que permitan innovar en el plano teórico y en la actividad profesional así como si existen propuestas que permitan que la Antropología pueda jugar un papel social más relevante» (Calvo, 2017: 191).

\section{A manera de conclusión: el legado de Claudio Esteva Fabregat y la antropología del siglo xxI}

Más allá de su legado científico, así como de su papel en la constitución de la antropología que hoy predomina en España, ¿cuáles son las enseñanzas que Esteva nos ha dejado para continuar y ampliar en la medida de las posibilidades el horizonte de esta disciplina? Desde mi punto de vista, dos aspectos fundamentales conforman su herencia, elementos que se encuentran en el centro de su pensamiento y de su configuración de la ciencia de la cultura y que, a la vez, conectan directamente con algunas de las contingencias actuales de nuestra sociedad y de nuestra disciplina. En primer lugar, la propia concepción holística de la antropología, de manera especial en la actualidad, cuando cada vez más se apela a la interdisciplinariedad. El ritmo del cambio social, así como los propios avances tecnológicos, están poniendo de manifiesto que, o bien la antropología reivindica su papel en conexión con otras disciplinas, como la antropología biológica y sus cada vez más destacados desarrollos en el ámbito de la genética de poblaciones, o bien su papel se verá muy reducido. Como simple muestra de ello, piénsese por un momento en el creciente discurso sobre nuestra base genética, que plantea que esta es la que conforma nuestras formas de vida socioculturales. Ello lleva a plantear, entre otras cosas, que las desigualdades —escolares, sociales...- ya vienen configuradas en nuestras bases genéticas, con todo lo que ello comporta. Así, véanse las tribunas aparecidas en los últimos meses en Le 
Monde sobre el papel de la genética y de la cultura en el desarrollo escolar infantil (vid. Atlan et alii, 2018; y Larregue, 2018), textos en los que, una vez más, se pone de manifiesto la instrumentalización pseudocientífica de los datos genéticos para explicar las diferencias psicológicas, sociales y culturales entre los seres humanos. Tal como he explicado en otro texto, en estos momentos la alianza de la antropología de carácter sociocultural con los nuevos avances en biología y genética de poblaciones es un campo que ofrece una amplia proyección para la ciencia de la cultura, ya que abre nuevos horizontes. Así, me pregunto, ¿cómo incorporar los nuevos instrumentos de análisis científico ( $\mathrm{ADN}$, análisis socioecológicos...) en la investigación antropológica de carácter cultural y social? ${ }^{33}$ ¿Quizás habría que recuperar la visión holística que Esteva planteó en el I Congreso Español de Antropología, celebrado en Barcelona en 1977?

En segundo término, otro de los aspectos centrales del pensamiento y de las preocupaciones de Esteva fue la antropología aplicada, tal como ya se ha apuntado más arriba cuando se ha hecho mención del proyecto que se presentó al IV Plan de Desarrollo. A la vista de algunas de las grandes cuestiones actuales, me pregunto: ¿cómo incorporar la antropología a los grandes desafíos que la humanidad tiene ante sí en este milenio, ya sean desde un enfoque natural (la era del Antropoceno) o cultural (ciudades globales-megalópolis, migraciones, refugiados, exclusión ...)? ¿Quizás la antropología del nuevo milenio está obligada a centrarse en la resolución de problemas? Estas cuestiones están en sintonía con la creciente preocupación de muchos antropólogos sobre la presencia pública de la disciplina en la actualidad, tal como se puso de manifiesto, por ejemplo, durante el seminario Making Anthropology Matter ${ }^{34}$, donde, como conclusión de una de las intervenciones, se pudo escuchar lo siguiente: «Since our strength lies in producing knowledge about phenomena that cannot easily be counted or measured, anthropologists have to make an effort to show the relevance of their irrelevant knowledge. Equally, if nobody

33 Tan solo a modo de muestra, vid. Bortolini et alii, 2017; «What knowledge societies can learn from foraging societies?»; y Johansson, 2003.

34 Seminario organizado por la EASA en colaboración con el Instituto de Etnología de la Academia Checa de Ciencias y la Asociación Checa de Antropología Social (Praga, 14 y 15 de octubre de 2015, vid. $<$ https://easaonline.org/agm.shtml>). 
understands what we are saying, that is not an indication of profundity but of poor language skills and muddled thought. As Marshall McLuhan once put it, even mud can give the illusion of depth. We can be sand the machinery, but we can also open up new vistas» (Eriksen, 2016).

Y, como última enseñanza de Esteva, cabe mencionar su continuada apelación a fortalecer la disciplina con nuevos instrumentos como, por ejemplo, mediante la creación de un colegio profesional: «Tot és un problema de recursos, però, mentrestant, la ja, diguem-ne, munió d'estudiants que la segueixen ha començat a produir la pressió que és necessària per a l'acolliment de la disciplina en forma d'una professionalitat no solament acadèmica, sinó també collegial» (Esteva, 2008: 74). Estos aspectos siempre habían ocupado un lugar destacado en sus preocupaciones, hasta el punto de que en 1982 afirmaba según sigue: «La dificultat més gran que nosaltres trobem és que no hi ha un mercat de treball institucionalitzat fora de la universitat. De manera que nosaltres, quan tenim un alumne que acaba els seus estudis, no podem dir-li que a fora de la universitat hi ha una demanda d'antropòlegs. No li podem dir perquè no existeix. Encara no existeix, dins del que és l'economia del país, una demanda institucionalitzada d'antropòlegs» (Hernández, 1982: 50). Estas declaraciones de 1982 coinciden con lo que otros profesionales han expresado en foros, reuniones y escritos. A mi juicio, son hoy un desafío que Esteva nos ha legado a todos los que trabajamos en pro de la antropología y que, en definitiva, deberían constituir un aldabonazo para propiciar un amplio debate sobre el presente y futuro de la disciplina, tanto en nuestras instituciones como en nuestra sociedad. 


\section{Bibliografía citada}

«Memoria de las actividades del Centro de Etnología Peninsular (19701972), de Barcelona». Ethnica 5 (Barcelona, 1973), p. 218.

«What knowledge societies can learn from foraging societies?». Recuperado de: <https://phys.org/news/2016-06-knowledge-societies-foraging.html>

Atlan, H. et alii 2018. «Halte aux 'fake news' génétiques», Le Monde. Publicado el 25/04/2018.

Bortolini, E.; Pagani, L.; Crema, E. R.; Sarno, S.; Barbieri, C.; Boattini, A.; Sazzini, M.; Graça da Silva, S.; Martini, G.; Metspalu, M.; Pettener, D.; Luiselli, D.; Tehrani, J. J. 2017. «Inferring patterns of folktale diffusion using genomic data». Proceedings of the National Academy of Sciences of United States of America -PNAS- 114(34): 9140-9145. Recuperado de: <http://www.pnas.org/content/114/34/9140>, doi: 10.1073 / pnas.1614395114

Calvo, L. 1996. «Claudio Esteva Fabregat, Éthnica y la Antropología», Ensayos de antropología cultural. Homenaje a Claudio Esteva-Fabregat. Barcelona: Ariel, 42-49.

CALvo, L. 1997. Historia de la antropología en Cataluña. Madrid: CSIC

CALvo, L. 2002a. «Éthnica. Revista de Antropología y su significación histórica para la antropología en España», Revista de Dialectología y Tradiciones Populares LVII(1): 71-82. Madrid: CISC.

CALvo, L. 2002b. Memoria del presente: la antropología española en sus publicaciones periódicas, número especial de la Revista de Dialectología y Tradiciones Populares LVII(1). Madrid: CSIC.

CALVo, L. 2017. «Del ayer, del hoy y del mañana de la antropología en Cataluña: en homenaje a Claudio Esteva Fabregat», Arxiu d'Etnografia de Catalunya 17:179-202. Tarragona: Publicacions URV.

CAPEL, H. 2009. «La antropología española y el magisterio de Claudio Esteva Fabregat. Estrategias institucionales y desarrollo intelectual en las disciplinas científicas», Scripta Nova. Revista Electrónica de Geografía y Ciencias Sociales XII (287). Barcelona: Universidad de Barcelona. Recuperado de <http://www.ub.es/geocrit/sn/sn-287.htm>. 
CASAS E. 1947. «Lecciones de dos maestros: Van Gennep y Corso», Revista de Dialectología y Tradiciones Populares III: 256-272. Madrid: CSIC.

Comas, D. / Pujades, J. J. 1975. «Elementos para un modelo del proceso urbanización/desruralización en el Alto Aragón», Ethnica 9: 37-74. Barcelona: CSIC.

Contreras, J. 1996. «Con el doctor Esteva en Chinchero (Perú). Aprendiendo el compadrazgo», Ensayos de antropología cultural. Homenaje a Claudio Esteva-Fabregat. Barcelona: Ariel, 36-41.

Eriksen, T. H. 2016. «Public Anthropology in the 21st Century, with Some Examples from Norway», Český lid: etnologický časopis (Český lid: Ethnological journal) 103(1): 23-36. Recuperado de <http://ceskylid.avcr. $\mathrm{cz} / \mathrm{medi} /$ articles/620/submission/original/620-1469-1-SM.pdf>.

Esteva, C. 1964. «El método funcionalista en el estudio de la aculturación», VIe Congrès International des Sciences Anthropologiques et Ethnologiques II. París: Musée de l'Homme, 583-587.

Esteva C. 1969. «La etnología española y sus problemas», I Congreso Nacional de Artes y Costumbres Populares. Zaragoza: Institución Fernando el Católico, 1-40.

Esteva, C. 1971. «Para una teoría de la aculturación en el Alto Aragón», Ethnica 2: 9-75. Barcelona: CSIC.

Esteva C. 1973a. «Aculturación y urbanización de inmigrados en Barcelona. ¿Cuestión de etnia o cuestión de clase?», Ethnica 5: 135-189. Barcelona: CSIC.

Esteva, C. 1973b. Antropología industrial. Barcelona: Planeta.

EsTEva, C. 1975. Razas humanas y racismo. Barcelona: Salvat.

Esteva, C. 1978. Cultura, sociedad y personalidad. Barcelona: Promoció cultural / Anthropos.

Esteva C. 1982. "Autobiografía intelectual»; Anthropos, Boletín de Información y Documentación 10: 4-18. Barcelona: Anthropos.

Esteva, C. 1984a. Estado, etnicidad y biculturalismo. Barcelona: Península.

Esteva, C. 1984b. «Final de etapa», Ethnica 20: 3-5. Barcelona: CSIC.

EsTeVA, C. 1993. «Antropología cultural», Diccionario temático de antropología. Barcelona: Boixareu, 26. 
EsTeVA C. 2008. «Elements per a una història de l'antropologia catalana», Construint ciència des de la Catalunya contemporània. Barcelona: Residència d'Investigadors, 32.

Frigolé, J. 1971. «Crónicas», Ethnica 2 (Barcelona, 1971), p. 232

Hernández, P. 1982. «Entrevista amb Claudi Esteva Fabregat», Ciència 16(2): 44-51. Barcelona.

JimÉNez, C. / CheCA, F. 2012. «Treinta años de antropología en España. Memoria desde la Gazeta». Gazeta de Antropología 28(3). Granada:Universidad de Granada. Recuperado de <http:/hdl.handle.net/10481/23747>.

Johansson, M. 2003. "Plenty of room at the bottom': Towards an anthropology of nanoscience», Anthropology Today 19(6). Recuperado de <https://doi.org/10.1111/j.0268-540X.2003.00228.x>.

Lagunas, D. 2012. «Claudio Esteva Fabregat, interpelado», Biblio 3W. Revista Bibliográfica de Geografía y Ciencias Sociales XVII(974). Barcelona: Universidad de Barcelona. Recuperado de <http://www.ub.edu/geocrit/ b3w-974.htm>.

Larregue, J. 2018. «Et Bourdieu dans tout ça ?» Le Monde. Publicado el 19/05/2018.

Lisón C. 2012. «Reminiscencias en levedad», Anthropos: Huellas del Conocimiento 235:27-36. Barcelona: Anthropos.

PRAT, J. 1992. Antropología y etnología. Madrid: Editorial Complutense y Caja de Ahorros y Monte de Piedad de Madrid.

PRAT, J. (coord.) 1999. Investigadores e investigados. Literatura antropológica en España desde 1954, monográfico del Arxiu d'Etnografia de Catalunya 4-5. Tarragona: Institut Tarragonès d>Antropologia.

PRAT, J.; Martínez, A. (eds.) 1996. Ensayos de antropología cultural. Homenaje a Claudio Esteva-Fabregat. Barcelona: Ariel.

VV. AA. 1980. I Congreso Español de Antropología. Actas. Barcelona: Universidad de Barcelona.

VV. AA. Anuario de la Universidad de Barcelona 1961-1962. Barcelona: Universidad de Barcelona.

VV. AA. Anuario de la Universidad de Barcelona 1967-1968. Barcelona: Universidad de Barcelona. 
VV. AA. Anuario de la Universidad de Barcelona 1968-1969. Barcelona: Universidad de Barcelona.

VV. AA. Anuario de la Universidad de Barcelona 1969-1970. Barcelona: Universidad de Barcelona.

VV. AA. Anuario de la Universidad de Barcelona 1971-1972. Barcelona: Universidad de Barcelona.

VV. AA. Anuario de la Universidad de Barcelona 1975-1976. Barcelona: Universidad de Barcelona.

VV. AA. Anuario de la Universidad de Barcelona 1977-1978. Barcelona: Universidad de Barcelona. 